\title{
Acute Effects of Ketamine on Memory Systems and Psychotic Symptoms in Healthy Volunteers
}

\author{
Celia JA Morgan*,', Ali Mofeez², Brigitta Brandner², Lesley Bromley² and H Valerie Curran' \\ 'Clinical Psychopharmacology Unit, Sub-Department of Clinical Health Psychology, University College London, London, England; ${ }^{2}$ UCL Centre for \\ Anaesthesia, University College London Hospital, London, England
}

\begin{abstract}
N-methyl-D-aspartate (NMDA) receptor antagonists have been demonstrated to induce schizophrenia-like symptoms and cognitive impairment in humans. The NMDA receptor has been strongly implicated in memory, but research to date on the effects of NMDA antagonists has examined only some aspects of human memory functions. This study used a double-blind, placebo-controlled, independent groups design with 54 healthy volunteers to examine the effects of infusions of two doses $(0.4,0.8 \mathrm{mg} / \mathrm{kg})$ of the NMDA antagonist ketamine upon the five human memory systems, aspects of executive functioning and schizophrenia-like and dissociative symptoms. Ketamine produced a dose-dependent impairment to episodic and working memory and a slowing of semantic processing. Ketamine also impaired recognition memory and procedural learning. Attention, perceptual priming and executive functioning were not affected following the drug. In addition, ketamine induced schizophrenia-like and dissociative symptoms, which were not correlated with the cognitive measures. These data suggest that, in humans, ketamine produces a selective pattern of impairments to working, episodic, and procedural memory but not to perceptual priming, attention or aspects of executive functioning.

Neuropsychopharmacology (2004) 29, 208-2 I8, advance online publication, 5 November 2003; doi: I0. I038/sj.npp. 1300342
\end{abstract}

Keywords: memory; glutamate; schizophrenia; N-methyl-D-aspartate receptors; ketamine; cognition

\section{INTRODUCTION}

Ketamine acts as an antagonist at glutamatergic $N$-methyld-aspartate (NMDA) receptors, which are found throughout the brain, but are most densely located in the hippocampus and cerebral cortex, areas important in human memory and cognition. Preclinical studies have shown that NMDAreceptor antagonists disrupt hippocampal long-term potentiation (LTP), the putative mechanism underlying neuronal learning (Morris et al, 1986). It has been proposed that NMDA-receptor-mediated LTP is involved in both working and long-term memory in humans (Lisman et al, 1998). Indeed ketamine, when administered to healthy volunteers, induces deficits in performance on verbal and visual memory tasks (Adler et al, 1998; Harborne et al, 1996; Harris et al, 1975; Hetem et al, 2000; Krystal et al, 1994, 1999, 2000; Malhotra et al, 1996).

Human memory research has been strongly influenced by the memory systems approach, developed by Tulving (1985, 1998). A memory system is proposed to comprise of 'a set of correlated processes' (Tulving, 1985). Distinct memory

*Correspondence: CJA Morgan, Clinical Psychopharmacology Unit, Sub-Department of Clinical Health Psychology, University College London, London WCIE 6BT, UK, Tel: +44 207679 1932, Fax: + 44 $2079 \mid 6$ 1989, E-mail: c.morgan@ucl.ac.uk

Received 20 March 2003; revised 05 August 2003; accepted 01 October 2003

Online publication: 06 October 2003 at http://www.acnp.org/citations/ Npp I0060303 | |8/default.pdf systems are seen to have different laws and principles characterizing their operation and differences in their ontogenetic and phylogenetic development. The five proposed memory systems are episodic memory, working memory, semantic memory, procedural memory, and the perceptual representation system (PRS).

Episodic memory makes possible what Tulving (1998) terms 'mental time travel' back into a person's past. It facilitates the acquisition and retrieval of information about specific personal experiences that occur at a particular time and place (Tulving, 1983). Episodic memory is thought to be recollection associated with a specific state of conscious awareness that allows one to 're-live' an event mentally ('remember'), as opposed to a feeling of familiarity but no conscious recollection of the event ('know') (Tulving, 1985). One task used to investigate episodic memory examines these 'remember' and 'know' responses following a presentation of stimuli. Another widely used episodic memory task tests memory for the 'source' of presented information, for example, remembering when, where or in which voice words were presented, in addition to examining memory for the words themselves. The semantic memory system encompasses a person's general knowledge about the world (Tulving, 1972) and, unlike episodic memory, semantic information is not associated with a specific learning context. Semantic knowledge covers a range of organized information including concepts, vocabulary, and facts. Semantic memory tasks include generating category members or judging whether sentences are true or false 
based on their semantic content. Working memory is thought to facilitate the maintenance and manipulation of internal representations that can be used to guide future behavior (Baddeley, 1998). Asking participants to repeat a list of digits backwards is considered a working memory task, as is requiring participants to judge whether, in a list of sequentially presented digits, one number is the same as the one presented immediately before it.

The memory system defined as the PRS can be viewed as a collection of domain-specific modules operating on perceptual information about the form and structure of words and objects. In memory research, the main interest in the PRS relates to its role in perceptual priming. Perceptual priming refers to an increased likelihood of identifying or recalling an object as a result of previous exposure to it. An example of a priming task would be one in which during the study phase, participants are presented with a list of words but they are not informed that these words will be used for a memory test. Then in the test phase, participants are asked to complete word fragments with the first word that comes to mind. In a priming task, some of these word fragments would be parts of words that have been previously presented in the study list. Participants would be expected to complete more fragments with previously presented words, without awareness of doing so. Finally, procedural memory pertains to the learning of motor and cognitive skills, examples of which are learning to ride a bike or to read. To test this experimentally, participants may be shown a series of numbers or letters in different locations in a set sequence, to which they have to respond as fast as they can. In the test phase, while unaware that they have been previously presented with a sequence, participants' reaction times (RTs) will nevertheless decrease when they are presented with numbers in the same order as those presented at study.

Previous research has addressed the effect of NMDA antagonism, via administration of ketamine, on some of these human memory systems. While several studies have found ketamine-induced impairments in verbal memory (eg Krystal et al, 1994; Harborne et al, 1996; Malhotra et al, 1996), only one study has investigated the effect of NMDAreceptor antagonism on the episodic memory system (Hetem et al, 2000). Both 'remember' and 'know' responses were reduced following a single dose of ketamine when compared to placebo. The authors argue that this indicates an impairment of episodic memory. A reduction in 'remember' responses supports this assertion but the reduction in 'know' responses may reflect semantic impairments. The effect of ketamine on the semantic memory system has been investigated in tasks in which participants are required to generate category members (semantic fluency) or words beginning with a specified letter (phonemic fluency). Fluency is generally considered to be a measure of frontal lobe function (Pendelton et al, 1982). However, neuroimaging studies have consistently implicated activation in the left prefrontal cortices during fluency tasks, which are areas activated in tasks requiring semantic retrieval (see Buckner, 2001). Hence deficits in verbal fluency may well reflect semantic memory deficits. Conflicting results have been obtained from ketamine studies with fluency tasks of both impaired (Adler et al, 1998) and preserved (Ghoneim et al, 1985) category fluency, and similar findings of an impairment to verbal fluency in some (Adler et al, 1998; Krystal et al, 1994, 1998), but not all (Krystal et al, 1999; Newcomer et al, 1999) studies. Findings concerning ketamine's effects on working memory are also conflicting. Impairments have been found on the $N$-back task (Adler et al, 1998), but not on a spatial working memory task (Newcomer et al, 1999). In addition, two studies have found preserved backwards digit span (Ghoneim et al, 1985; Harris et al, 1975), but one study has found impaired forwards digit span (Harris et al, 1975). Newcomer and Krystal (2001), in their review of memory research with NMDA antagonists thus far, noted that ketamine's effects on memory and learning appear to be preferential to their effects on other cognitive functions and may be dose dependent. However, the effects of ketamine on the other two memory systems, procedural learning and perceptual representation, have not previously been investigated.

Although several studies have investigated the acute cognitive effects of ketamine on memory, they have been confined to single doses or, where more doses have been used, crossover designs have complicated the interpretation of results due to the tachyphylaxis (or rapidly developing tolerance) that occurs following ketamine administration. Further, previous research has not examined the effect of this drug on the full range of human memory functions.

Therefore, the present study aimed to characterize thoroughly the impact of ketamine administration on the different memory systems using a dose-response methodology. It was hypothesized that ketamine administration would impair episodic and working memory, replicating and extending previous findings. By using a more specific semantic memory task, this study hoped to clarify the effect of ketamine on this memory system. No prediction could be made as to the effects of ketamine on procedural learning or perceptual priming as these have not previously been investigated. The subjective effects that ketamine was expected to induce were increases in dissociative and schizotypal symptomatology.

\section{METHODS}

\section{Participants and Design}

Participants were recruited through an advertisement and were paid for their participation. The study was carried out in accordance with the Declaration of Helsinki and was approved by the institutional ethics committee (the UCL/ UCLH Committee on the Ethics of Human Research). All participants gave written, witnessed, informed consent. The inclusion criteria were that participants were between 18 and 35 years old and native English speakers. Participants were then selected for participation by a screening interview to exclude individuals with a propensity towards psychiatric disorders, and any substance misuse or general health problems.

Volunteers were screened by a semi-structured interview (conducted by CM), questionnaire, and physical examination to exclude those with (i) psychotic illness in a firstdegree relative; (ii) current or past psychiatric problems: the Beck Depression Inventory, (Beck, 1978) and Speilberger Trait Anxiety Inventory, (Spielberger, 1983) were used to 
screen current psychopathology in addition to DSM IV diagnostic criteria for psychotic illnesses; (iii) any significant history of substance misuse, including prior ketamine use; (iv) a positive result in a urinalysis screening for drugs of abuse; (v) hypertension; (vi) outside normal levels on the body mass index; (vii) a known allergy to ketamine; (viii) taking any prescribed medication (excluding oral contraceptives). At the screening interview, the premorbid IQ of each participant was also assessed by the 'Spot the Word' test (Baddeley et al, 1993), an estimate of verbal intelligence based on lexical decision; trait dissociation was assessed by the Dissociative Experiences Scale (DES, Bernstein and Putnam, 1986).

A total of 69 volunteers responded to the advertisement and 11 were excluded for failing to meet the inclusion criteria (history of mental illness - two, family history of mental illness - three, prior use of ketamine-three, currently on medication - three). In all, 58 healthy volunteers took part in this study. Of these, four participants dropped out: two had adverse reactions to cannulation, one had an adverse reaction to $0.8 \mathrm{mg} / \mathrm{kg}$ ketamine and did not wish to continue with the experiment, and one participant was excluded due to failing a urine screen upon the followup testing day. In total, 54 participants completed the study (mean age $22.53 \pm 3.52$ years). A mixed model, independent group design was used, in which male and female participants were randomly allocated to treatment with either $0.8 \mathrm{mg} / \mathrm{kg}$ ketamine hydrochloride, $0.4 \mathrm{mg} / \mathrm{kg}$ ketamine hydrochloride, or placebo. Groups were balanced for gender with nine females and nine males in each group. Double-blind procedures were used throughout. Test versions were counterbalanced across participants and design.

\section{Drug Administration}

Participants received either ketamine $(0.4$. or $0.8 \mathrm{mg} / \mathrm{kg}$ ) or saline placebo $(0.9 \% \mathrm{NaCl})$ intravenously for $80 \mathrm{~min}$. Pilot work demonstrated that tasks were sensitive to these doses of ketamine. Low doses were chosen to ensure that participants would understand the instructions to the tasks and to minimize adverse effects. This produces a dose of 0.005 or $0.01 \mathrm{mg} / \mathrm{kg} / \mathrm{min}$ ketamine in the low- and high-dose groups. Using local anesthesia, a 16-gauge intravenous cannula was inserted in the nondominant forearm and after 5 min the ketamine infusion began via a Graseby intravenous infusion pump. A loading dose was not used in order to minimize adverse effects. A urine sample was taken before the infusion began and a peripheral venous blood sample was taken $65 \mathrm{~min}$ after commencing the infusion. Plasma was obtained immediately from blood samples by centrifugation and samples were stored at $-80^{\circ} \mathrm{C}$. Ketamine levels were measured using gas chromatography (ABS Laboratories, National Poisons Unit, London).

\section{Procedure}

Testing occurred at either 0900 or 1300 and the time of testing was broadly matched across groups. Participants arrived at the hospital after completing an overnight fast for morning testing, or a minimum of $6 \mathrm{~h}$ fasting for afternoon testing. Participants were assessed on the predrug battery for $35 \mathrm{~min}$. They were then allowed to rest for $15 \mathrm{~min}$ and were then cannulated. Approximately $5 \mathrm{~min}$ after cannulation, the anesthetist began the infusion. Participants were tested on a battery of tests similar to the predrug battery beginning $20 \mathrm{~min}$ after the start of infusion. Throughout the 80 min infusion the participant's pulse, blood pressure, and electrocardiagram were monitored. After infusion, participants were provided with light refreshments and were then assessed $30 \mathrm{~min}$ later and then at hourly intervals by the medical staff as to their 'street readiness'. A follow-up battery was given 3 days after the acute dose, findings of which are reported elsewhere (Morgan et al, in press).

\section{Assessments}

Tests were selected to assess the range of human memory functions, dissociative and psychotogenic symptoms, and mood effects. Tests were administered in the following order: subjective drowsiness, speed of comprehension, serial reaction time (SRT) task, trailmaking, word-stem completion, and cued recall, $N$-back working memory task, source memory, CADDS SSQ, subjective drowsiness.

\section{Cognitive Tasks}

Speed of comprehension (Baddeley et al, 1992). This task taps verbal semantic memory. Participants were presented with 200 sentences, some of which made sense (eg 'Sharks are good swimmers') and some of which do not (eg 'Wives are made in factories'). They were given 2 min to mark which sentences made sense and which ones did not. The task was scored in number of sentences completed and number of errors.

Source memory task (Wilding and Rugg, 1996). This task was chosen as an index of episodic memory, that is, awareness of when and where a stimulus was encoded. Stimuli consisted of 240 low-frequency words. The words were divided randomly into six study lists of 40 words. In each study list, half the words were spoken in a female voice and half in a male voice (allocation was randomly determined). At study, words were presented to participants aurally, played on a tape recorder. During the study phase, participants listened to each word, repeated it aloud and then, depending upon the gender of the voice it is presented in, rated the word as either 'pleasant/unpleasant' or 'abstract/concrete.' After completing the list, there was a delay of $6 \mathrm{~min}$, filled with another task, and then participants were presented with a test list. Test lists were created by combining the study list with another study list that had not been presented. Test words were presented visually on a computer monitor. Participants were instructed to say aloud whether each word was one that they had heard before and if so, whether it had been presented in a male or female voice. Participants gave their responses verbally. Word recognition responses were recorded as hits, false alarms, misses, and correct rejections. Source errors were also recorded.

Word-stem completion and cued recall (Bishop and Curran, 1995). This task was chosen as an index of perceptual priming and free recall, using a levels of 
processing (LOP) encoding task (to distinguish between the perceptual representation system, which should not be affected by LOP, and the episodic memory system, which should be affected by LOP). Stimuli were 96 words. In the study phase, the participants were required to read aloud 64 words presented on the computer screen each for $4000 \mathrm{~ms}$ with a $1500 \mathrm{~ms}$ interval. For each block of 32 words, the participants were given encoding instructions. For the semantic encoding condition, participants were instructed to say whether the word presented depicted something living or nonliving. For the physical encoding condition, participants were instructed to say how many vowels were in each of the words. The encoding condition was counterbalanced across treatment groups. In the test phase, the word-stem completion task was given before the cued recall. In the word-stem completion task, participants were presented with 64 word-stems (16 previously semantically encoded, 16 previously physically encoded, 32 unseen) and asked to complete them with the first word that 'popped into their head', excluding proper nouns. Afterwards, participants were asked if they noticed anything about the words that they had completed the word-stems with. The cued recall task then followed. Participants were given a sheet of 32 word-stems (16 semantically encoded, 16 physically encoded) and were told that they had previously been shown all of the words that completed the word-stems. Participants were then asked to complete the stems with words they remembered being shown on the computer screen. Scores were recorded in terms of completion rates across the two encoding conditions and errors in the cued recall task and noncompleted stems in the word-stem completion.

N-back working memory task (Braver et al, 1997). The task used a sequential letter paradigm and manipulated working memory load incrementally. The ' 0 -back' condition was taken as an index of attention and the '1-back' and '2back' conditions as tapping working memory. Stimuli were sequences of lower case consonants presented centrally for $1200 \mathrm{~ms}$ with a $500 \mathrm{~ms}$ interstimulus interval. Stimuli were organized in a pseudorandom sequence with targets occurring on $33 \%$ of the trials. Blocks were of 112 seconds (66 stimuli); each participant experienced one block in each condition at each time point (total nine blocks). Participants were presented stimuli on a VDU and responded to stimuli with their dominant hand, pressing a 'yes' button for targets and a 'no' button for nontargets. In the ' 0 -back' condition, participants were required to respond 'yes' if they saw a target letter (eg M). In the 1-back condition, participants responded 'yes' to a letter if it was the same as the letter before it, in the 2-back condition if a letter was the same as two before it. RTs and responses were recorded for each trial using Visual Basic software.

Trailmaking (Reitan, 1958). This task consisted of two parts, the first tapping psychomotor speed and the second executive functioning. In Part A of the task, participants were required to connect 25 circles in ascending order as rapidly and accurately as possible. Part B again contains circles, but with both numbers and letters in ascending order. Participants are again required to connect the circles as rapidly as possible, this time alternating between the two different sequences ( 1 to $\mathrm{A}, \mathrm{A}$ to 2,2 to $\mathrm{B}, \mathrm{B}$ to 3 , etc). A difference score was computed by taking time to complete on Part A away from time on Part B to give a measure of executive functioning controlling for simple psychomotor speed. Errors were also recorded.

SRT task (Shanks and Perruchet, 2002). This task was used as an index of procedural learning using a repeating sequence. Participants were required to press a key as soon as they see a target appear in one of the four boxes on the computer screen. Participants are told that the task is a simple RT experiment, but actually the targets appear in boxes in a set sequence, for $85 \%$ of the trials. The task thus taps participants' ability to learn this underlying sequence. After a practice block of 10 trials with no underlying sequence, participants were subjected to one of two sequences balanced across conditions, for three blocks of 100 trials (a total of 300 trials). In each trial, a target (a ' $X$ ') appeared in one of the boxes. On $85 \%$ of the trials in each block, the ' $\mathrm{X}$ ' would be a location correspondent to the sequence that the participant was being trained on (probable trial). However, on $15 \%$ of the trials in each block, the ' $\mathrm{X}$ ' would appear in a location erroneous to the underlying sequence (improbable trial). The order of occurrence of the improbable trials was randomly determined. A trial ended when the participant pressed the correct corresponding key, whereupon the target moved to a new location. RTs were recorded with Visual Basic software, and scored in terms of latency of responding correctly for probable trials, latency of responding correctly for improbable trials, number of errors on probable trials, and number of errors on improbable trials.

\section{Subjective Ratings}

Schizotypal Symptomatology Questionnaire (Curran and Morgan, 2000): a 30-item questionnaire designed to assess state schizophrenic-like symptoms in normal populations.

Dissociative. States Scale (adapted from Bremner et al, 1998): a 19-item measure subjectively rated measure tapped state dissociative symptoms.

Mood Rating Scale (Bond and Lader, 1974): a six-item visual analogue scale (VAS) was used to investigate subjective drowsiness.

At the end of the main session, the effectiveness of blinding was also assessed, with both the participant and experimenter guessing whether they thought that a drug had been administered.

\section{Statistical Analyses}

All statistical analyses were performed using SPSS Version 9.0. Group differences were examined using one-way ANOVAs and, where data were nonparametric, the Kruskall-Wallis test. The recognition memory component of the source memory task and the $\mathrm{N}$-back were analyzed using the signal detection theory (Snodgrass and Corwin, 1988). This method was selected as it allows a separation of the response bias component from discriminability and yields a measure not only of the ability to recognize a word/number but also the bias in responding. Within psychopharmacological research response bias can be an issue, either in 
terms of disinhibition or overinhibition. Thus it is important to separate response bias, where possible, from memory data. The recognition and $N$-back data were then, along with most other cognitive tasks and subjective effects, analyzed using $3 \times 2$ repeated measures analyses of variance (RMANOVA) with time (predrug, postdrug) as the withinsubject factor and drug condition (placebo, $0.4 \mathrm{mg} / \mathrm{kg}$ ketamine, $0.8 \mathrm{mg} / \mathrm{kg}$ ketamine) as the between-subject factor. Where significant interactions on $2 \times 3$ were found, one-way ANOVAs on change scores between pre- and postdata were conducted, followed by orthogonal contrasts comparing (1) placebo with both drug groups and then (2) low- and high-dose ketamine. Dunnett's $t$ and simple effects were analyzed in RMANOVAS with more than two factors. Bonferroni corrections were used to control for multiple comparisons and correlations.

\section{RESULTS}

\section{Trait Scores, Demographics and Drug Dosage}

There were no significant group differences in age. Participants were additionally matched in a premorbid IQ (spot the word) test, depression, alcohol and tobacco use, and trait dissociation. There were no differences in the milliliters infused for the three groups $(\mathrm{F}(2,51)=0.39$, $p=0.68)$ or between the weights of the groups $(\mathrm{F}(2,51)=0.44, p=0.65)$ (Table 1$)$. In total, the 0.8 and $0.4 \mathrm{mg} / \mathrm{kg}$ groups received a mean of $56.45 \pm 9.19$ and $26.74 \pm 7.56 \mathrm{mg}$ ketamine, respectively, over $80 \mathrm{~min}$.

\section{Cognitive Tasks}

Source memory. For recognition memory data $d^{\prime}$, an index of discriminability, and $C$, a measure of bias, were calculated. RMANOVA analysis of discrimination (revealed a significant drug $\times$ time interaction $(F=(2,51)=8.74$, $p<0.001)$ and significant main effects of time $(\mathrm{F}(1,51)=42.59, \quad p<0.001)$ and drug $(\mathrm{F}(2,51)=3.84$, $p<0.05$ ) (see Figure 1a). Post hoc analysis revealed significantly lower recognition memory scores in the drug groups as compared to placebo $(t(51)=3.98, p<0.001)$.

Analysis of the criterion $(C)$, found a significant time $\times$ drug interaction $(\mathrm{F}(2,51)=3.75, p<0.03)$ and a main effect of time $(\mathrm{F}(1,51)=21.92, p<0.001)$. Contrasts revealed a difference in bias between the $0.8 \mathrm{mg} / \mathrm{kg}$ and $0.4 \mathrm{mg} / \mathrm{kg}$ ketamine groups, such that the low-dose group was significantly less conservative than the higher dose group $(t(51)=-2.09 p<0.04)$. The criterion only changed in the high-dose group from $0.40 \pm 0.22$ predrug to $0.78 \pm 0.42$ postdrug, indicating that they became more conservative, that is, they were more likely to say that they had not heard a previously presented word. Data on the probability of a correct source judgment $(\mathrm{Ht})$ given recognition $\left(\mathrm{Ht}^{\prime}\right)$ were analyzed $\left(\mathrm{Ht} / \mathrm{Ht}^{\prime}\right)$. There was a significant interaction between time and drug $(\mathrm{F}(2,51)=18.84, p<0.001)$ and significant main effects of drug $(\mathrm{F}(2,51)=11.72, p<0.001)$ and time $(\mathrm{F}(1,51)=44.30, p<0.001)$. Contrasts found significantly lower correct identification of the source of memories in the drug groups as compared to placebo $(t(51)=4.91, p<0.001)$ and in the $0.8 \mathrm{mg} / \mathrm{kg}$ group compared to the $0.4 \mathrm{mg} / \mathrm{kg}$ ketamine group $(t(51)=3.68$, $p=0.001)$.The clear dose-response relationship can be seen in Figure 1b. However, even given the marked impairments of the high-dose group compared to the low-dose and placebo groups, it is noteworthy that the high-dose ketamine group was still performing above the 0.5 chance level.

Speed of comprehension. There was a significant drug $\times$ time interaction $(\mathrm{F}(2,51)=11.72, p<0.001)$. and main effect of time $(\mathrm{F}(1,51)=4.93, p<0.03)$ on the number of completed sentences. Contrasts revealed that the placebo group completed more sentences than the 0.8 and $0.4 \mathrm{mg} / \mathrm{kg}$ ketamine group $(t(51)=4.03, p<0.001)$, and significantly more sentences completed in the low-dose ketamine compared to the high-dose group $(t(51)=2.68$, $p=0.01)$. There was no effect of drug on the number of errors in the silly sentences task or any interaction (Figure 2).

$\mathrm{N}$-back working memory task. For the N-back working memory data $d^{\prime}$ and $C$ were also calculated. Analysis of $d^{\prime}$ with a $3 \times 2 \times 3$ ANOVA yielded a significant time $\times$ drug interaction $(\mathrm{F}(2,51)=7.49, \quad p<0.01)$ and a significant time $\times$ working memory load interaction $(\mathrm{F}(2,102)=3.69$, $p<0.03)$ in addition to main effects of working memory load $(\mathrm{F}(1,51)=350.12, p<0.001)$, time $(\mathrm{F}(1,52)=10.18$,

Table I Demographics Across Treatment Groups

\begin{tabular}{lccc}
\hline & Placebo, mean (SD) & $\begin{array}{c}\mathbf{0 . 4} \text { mg/kg ketamine, } \\
\text { mean (SD) }\end{array}$ & $\begin{array}{c}\mathbf{0 . 8} \text { mg/kg ketamine, } \\
\text { mean (SD) }\end{array}$ \\
\hline Age & $21.83( \pm 3.15)$ & $21.17( \pm 1.69)$ & $24.17( \pm 4.53)$ \\
Spot the word test score & $50.00( \pm 3.20)$ & $49.67( \pm 3.65)$ & $49.65( \pm 3.87)$ \\
Alcohol use (U/week) & $14.22( \pm 8.89)$ & $11.83( \pm 7.67)$ & $\mid 3.61( \pm 8.51)$ \\
Tobacco use (cigarettes/day) & $2.11( \pm 4.43)$ & $3.33( \pm 4.85)$ & $1.78( \pm 4.77)$ \\
BDI score & $3( \pm 3.6)$ & $4.3( \pm 4.9)$ & $3.5( \pm 4.9)$ \\
STA score & $34.6( \pm 10.8)$ & $32.5( \pm 8.79)$ & $33.8( \pm 9.7)$ \\
DES score & $32.3( \pm 32.39)$ & $31.6( \pm 30.3)$ & $31.5( \pm 35.5)$ \\
Weight (kg) & $71.29( \pm 13.92)$ & $66.84( \pm 18.87)$ & $70.56( \pm 11.49)$ \\
Milliliters infused & $31.2( \pm 6.05)$ & $29.8( \pm 3.71)$ & $30.9( \pm 5.58)$ \\
\hline
\end{tabular}



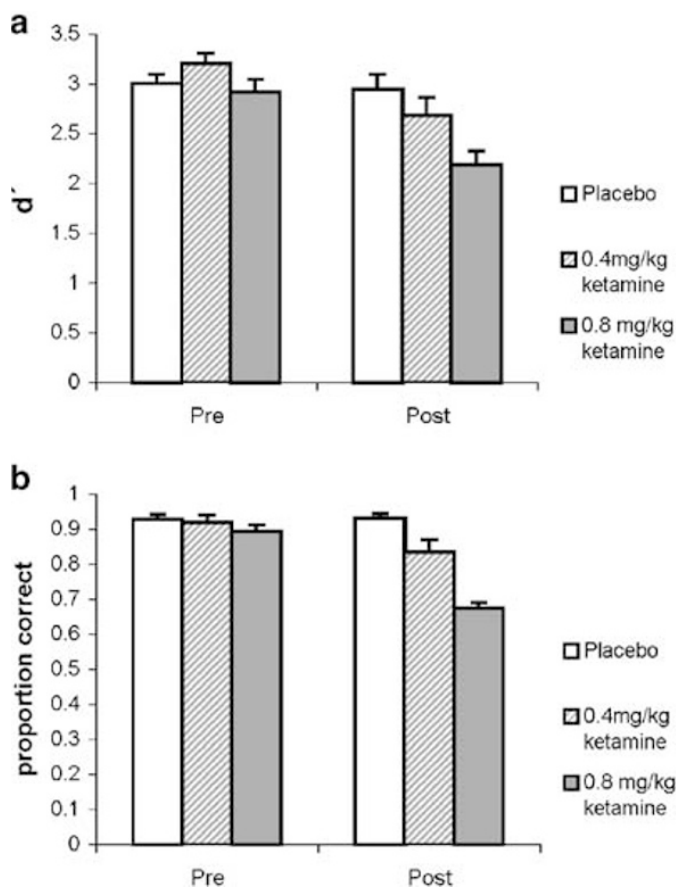

Figure I (a) Mean $d^{\prime}$ index for recognition memory at each assessment point by each treatment condition. (b) Mean proportion of correct source memory judgments pre- and postdrug across each condition.

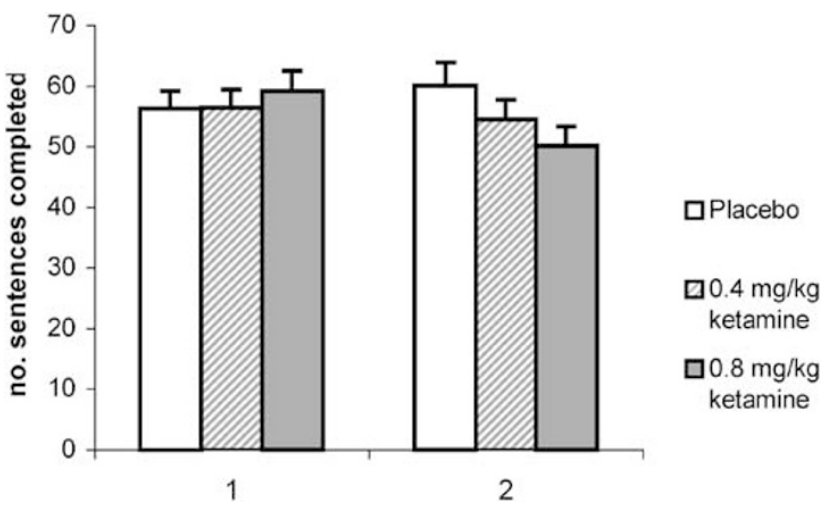

Figure 2 Mean number of sentences completed pre- and postinfusion across each treatment condition for speed of comprehension. $p<0.01)$, and drug $(F(2,51)=4.08, \quad p<0.03)$. Further analysis of this data demonstrated group differences postdrug on the 1-back $(\mathrm{F}(2,53)=5.58, p<0.01)$ and 2 back tasks $(\mathrm{F}(2,53)=6.32, p<0.01)$ only.

Contrasts indicated that these difference were attributable to lower scores in the drug groups compared to placebo on both the '1-back' $(t(51)=3.71, p=0.001)$ and '2-back' $(t(53)=2.34, p<0.03)$ tasks and lower scores in the 0.8 than the $0.4 \mathrm{mg} / \mathrm{kg}$ ketamine group, again both on the 1 back $(t(53)=2.25, \quad p<0.03)$ and 2-back $(t(53)=2.71$, $p<0.01)$. As can be seen from Figure 3, the high-dose ketamine group was performing at levels close to chance in the 2-back condition postdrug. The response criterion $(C)$ was also analyzed. A working memory load $\times$ drug interaction was found $(\mathrm{F}(4,102)=6.00, p<0.001)$, and a working memory load $\times$ time interaction $(\mathrm{F}(2,102)=189.414$, $p<0.001)$ in addition to main effects of working memory load $(\mathrm{F}(2,102)=110.21, p<0.001)$, time $(\mathrm{F}(1,51)=373.41$, $p<0.001)$. Differences between groups were revealed postdrug on the 2-back section of the task $(\mathrm{F}(2,53)=7.61$, $p<0.01)$. The drug groups were less conservative overall than the placebo group $(t(53)=3.07, p<0.01)$, and the high-dose group was less conservative than the low-dose group $(t(53)=2.45, p<0.02)$ (group means, postdrug were: placebo: $0.014 \pm 0.25 ; 0.4 \mathrm{mg} / \mathrm{kg}$ ketamine: $-0.17 \times 0.28$; $0.8 \mathrm{mg} / \mathrm{kg}$ ketamine: $-0.34 \pm 0.27)$. RTs were not found to differ between the groups at any of the time points. There was, however, a main effect of working memory load $(\mathrm{F}(1,51)=147.24, \quad p<0.001)$. Within subjects contrasts revealed RTs to increase with increasing memory load $(\mathrm{F}(1,43)=199.73, p<0.001)$.

\section{LOP Retrieval Intentionality (Table 2)}

Word-stem completion. Participants completed more wordstems with studied than unstudied words, that is, priming occurred, with a main effect of study $(\mathrm{F}(1,53)=50.47$, $p<0.001)$. The ratio of targets to distractors completed was computed as an index of priming. RMANOVA of these scores for semantically encoded stems and physically encoded stems revealed no effects of drug or LOP on priming.

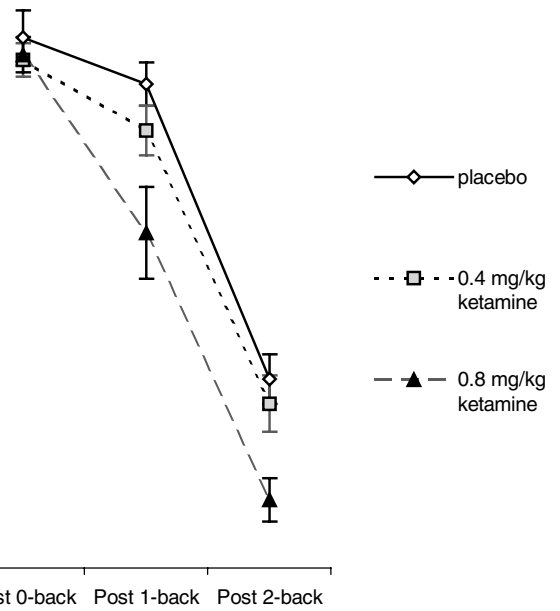

Figure 3 d' N-back working memory scores across working memory load, assessment point, and treatment condition. 
Table 2 Group Means and SDs for (Left to Right): Targets Completed, Distractors Completed, Ratio of Targets Completed to Distractors Completed, Targets Recalled

\begin{tabular}{|c|c|c|c|c|}
\hline Treatment & Targets completed & Distractors completed & Targets: distractors & Target recall \\
\hline \multicolumn{5}{|l|}{ Placebo } \\
\hline Overall & $12.44 \pm 2.77$ & $7.56 \pm 2.47$ & I.86: I ( \pm 794$)$ & $8.56 \pm 4.36$ \\
\hline Semantic & $6.17 \pm 2.24$ & & & $5.06 \pm 2.48$ \\
\hline Physical & $6.28 \pm 1.82$ & & & $3.50 \pm 2.44$ \\
\hline \multicolumn{5}{|c|}{ Low-dose ketamine } \\
\hline Overall & $9.94 \pm 2.36$ & $6.22 \pm 2.21$ & I.94: I ( \pm 1.37$)$ & $7.61 \pm 4.5$ \\
\hline Semantic & $4.44 \pm 2.73$ & & & $4.50 \pm 2.38$ \\
\hline Physical & $5.50 \pm 2.46$ & & & $3.11 \pm 2.78$ \\
\hline \multicolumn{5}{|c|}{ High-dose ketamine } \\
\hline Overall & $10.27 \pm 3.75$ & $7.56 \pm 2.48$ & I.63: I ( \pm 1.17$)$ & $5.33 \pm 3.61$ \\
\hline Semantic & $5.39 \pm 2.83$ & & & $3.33 \pm 2.30$ \\
\hline Physical & $4.88 \pm 2.11$ & & & $2.00 \pm 1.94$ \\
\hline
\end{tabular}

Cued recall. Unlike the word-stem completion condition, L-O-P (semantic vs physical) had a significant effect on stem cued recall $(\mathrm{F}(1,51)=19.53, p<0.001)$, with semantically encoded words being better recalled than physically encoded words. There was a trend for a main effect of drug $(\mathrm{F}(2,51)=2.83, p=0.068)$.

SRT task. The two sequences which participants were trained on were combined in the analysis. RTs are only used for trials on which participants did not make errors and RTs from the first two trials of each block were disregarded because it would be impossible for participants to predict the location of the next target. A $3 \times 2 \times 3$ RMANOVA found a significant block $\times$ drug interaction $(\mathrm{F}(4,106)=3.55, p<0.01, p=0.009)$, significant main effects of probable/improbable $(\mathrm{F}(1,53)=13.51, p<0.01)$ and drug $(\mathrm{F}(2,53)=8.59, p<0.01)$. Post hoc Dunnett's $t$ revealed the placebo group to be significantly faster than the $0.4 \mathrm{mg} /$ $\mathrm{kg}$ ketamine group $(p<0.02)$ and the $0.8 \mathrm{mg} / \mathrm{kg}$ group $(p<0.001)$. Multiple comparisons (with applied Bonferroni correction) revealed significant differences between probable and improbable trials for the placebo group at Block 2 $(t(18)=2.97, p<0.01)$ and Block $3(t(18)=3.52, p<0.01)$, but not at Block 1. For the $0.4 \mathrm{mg} / \mathrm{kg}$ ketamine group there were no differences, but at Block 3 the difference approached significance $(t(17)=2.28, p=0.036)$. There were no differences between the probable and improbable trials on any block in the $0.8 \mathrm{mg} / \mathrm{kg}$ condition. As seen in Figure 4, only the placebo group showed faster RTs (ie learning) over blocks; $0.4 \mathrm{mg} / \mathrm{kg}$ showed a flat curve and $0.8 \mathrm{mg} / \mathrm{kg}$ were slower over blocks. Analysis of the mean number of errors per block yielded a significant effect of condition $(\mathrm{F}(1,53)=6.12, p<0.02)$ and a significant main effect of block $(\mathrm{F}(2,106)=4.38, p<0.02)$. However, multiple comparisons did not demonstrate any further differences.

Trailmaking. For the time taken to complete part A, there were no significant group differences, although there was a

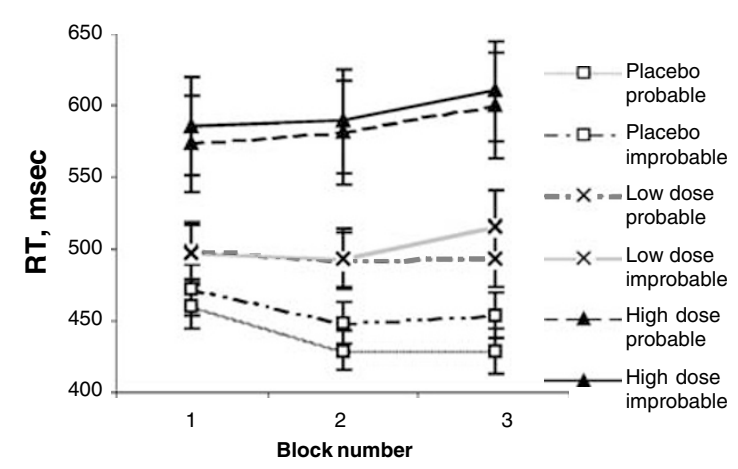

Figure 4 Mean RTs for the SRT task across probable and improbable trials.

trend for a time $\times$ drug interaction $(\mathrm{F}(2,51)=2.65$, $p=0.08)$. In part $\mathrm{B}$, there was a significant $\operatorname{drug} \times$ time interaction for the time taken to complete the task $(\mathrm{F}(2,51)=5.73, p<0.01)$. Contrasts demonstrated were greater time taken to complete the task by the two drug groups compared to placebo $(t(51)=2.50, p<0.02)$ and in the $0.8 \mathrm{mg} / \mathrm{kg}$ ketamine group compared to the $0.4 \mathrm{mg} / \mathrm{kg}$ ketamine group postdrug $(t(51)=2.81, p<0.01)$. An overall score for trailmaking was computed by subtracting the scores from the first part of the test from scores on the second part of the test. This was to remove any general motor impairment effects from the task and concentrate upon the executive functioning component. Overall, no significant effects of treatment condition, assessment point, or errors were observed.

\section{Subjective Effects}

Schizotypal Symptomatology data for schizotypy were not normally distributed, so were transformed by a square root transformation. However, this did not affect the results of 
the RMANOVAs so untransformed statistics and means are reported. When the overall score for schizotypy was computed, RMANOVA demonstrated a significant drug $\times$ time interaction $(\mathrm{F}(2,51)=10.95, p<0.001)$ and a significant effect of time $(F(1,51)=23.26, p<0.001)$. Contrasts showed that both the drug groups scored more highly than the placebo group $(t(51)=-4.63, p<0.001)$, but there were no differences between the low- and $0.8 \mathrm{mg} / \mathrm{kg}$ ketamine groups. Group means on this scale postdrug were $4.61 \pm 4.49$ in the placebo group, $14.39 \pm 14.56$ in the lowdose group, and $18.61 \pm 9.99$ in the high-dose group.

Dissociative states scale. RMANOVA of the overall DSS score showed a significant drug $\times$ time interaction $(\mathrm{F}(2,51)=21.30, p<0.001)$. and significant main effects of drug $(\mathrm{F}(2,51)=17.28, p<0.001)$ and time $(\mathrm{F}(1,51)=85.63, p<0.001)$. Ketamine induced clear, doserelated dissociative effects. These were confirmed by further analysis of the interaction, which revealed significantly lower scores in the placebo group compared to the two drug groups postdrug $(t(51)=-5.88, p<0.001)$ and the $0.4 \mathrm{mg} /$ $\mathrm{kg}$ ketamine group compared to the $0.8 \mathrm{mg} / \mathrm{kg}$ ketamine group postdrug $(t(51)=-2.85, p<0.01 p=0.003)$. Group means on the dissociative states scales postdrug were $2.33 \pm 3.20$ for the placebo group; $15.28 \pm 11.23$ for the low-dose group, and $27.28 \pm 15.91$ for the high-dose group.

Subjective drowsiness. A $3 \times 4$ RMANOVA of the visual analogue scale for 'drowsiness' yielded a significant drug $\times$ time interaction $(\mathrm{F}(6,153)=7.90, p<0.001)$, a significant main effect of drug $(\mathrm{F}(2,51)=9.22, p<0.001)$ and time $(\mathrm{F}(3,153)=67.93, p<0.001)$. Contrasts revealed that the placebo group were less drowsy than the two drug groups $10 \mathrm{~min}$ postdrug $(t(51)=-5.30, p<0.001)$, and 80 min postdrug $(t(51)=-6.19, p<0.001)$.

Guess on treatment. The high-dose ketamine groups were all accurate in discriminating the drug from placebo, and 16 $(89 \%)$ of the low-dose participants could discriminate the ketamine from placebo. The experimenter guessed incorrectly as to the treatment condition (placebo/low dose/high dose) 20 times (high-dose group 8/18 times, low-dose group $8 / 18$ times, placebo $4 / 18$ times), thus the experimenter was accurate $63 \%$ of the time.

Sex differences. No drug $\times$ gender interactions emerged on the source memory task, speed of comprehension, and the schizotypal symptomatology questionnaire.

Plasma ketamine levels at $65 \mathrm{~min}$. Mean plasma ketamine levels were $128.96 \mathrm{ng} / \mathrm{ml}( \pm 36.96)$ for the low-dose group and $261.90 \mathrm{ng} / \mathrm{ml}( \pm 31.56)$ for the high-dose group.

Other responses. Seven participants reported feeling nauseous (high dose - two males, four females, low dose -one male). Of these, one low-dose male participant vomited $5 \mathrm{~m}$ after the infusion had been stopped. However, all participants, when asked, felt able to continue with the tasks. Many participants in the ketamine groups reported visual changes such as blurred vision or a sense that everything was moving or flickering. Despite this, however, all participants felt that they were able to proceed with the tasks.

\section{DISCUSSION}

The present study investigated the effects of two doses of ketamine, 0.4 and $0.8 \mathrm{mg} / \mathrm{kg}$, on memory systems, and dissociative and schizotypal symptoms. The main findings were that ketamine impaired working and episodic memory with increasing dose, disrupted procedural, and semantic memory regardless of dose while leaving perceptual priming intact. Ketamine also induced schizophrenic and dissociative symptoms, replicating previous studies (Krystal et al, 1994; Malhotra et al, 1996; Newcomer et al, 1999; Adler et al, 1998; Hetem et al, 2000).

Ketamine dose dependently increased both source and recognition memory errors. These findings replicate the impairment in recognition memory observed previously with ketamine (Ghoneim et al, 1985; Malhotra et al, 1996; Hetem et al, 2000), but extend these findings to demonstrate an episodic memory impairment. The only previous study to examine episodic memory following ketamine indicated a decrease in both recollection and familiarity (Hetem et al, 2000). There clearly is a parallel between impairments to conscious awareness ('remembering') and the source memory impairments we observed. Our finding of source memory deficits indicates not only an impairment of 'what' is remembered but also 'how, why, and where'. This supports the notion that the NMDA receptor is important in episodic memory in humans. Neuroimaging studies suggest that these deficits in source memory may be a function of hyperactivation of the prefrontal cortex and underactivation of the hippocampus and medial temporal lobe structures, as these areas are associated with source memory (Wheeler et $a l, 1997$ ) and this pattern of neural activity has been found following ketamine administration (Breier et al, 1997; Vollenweider et al, 1997),

Interestingly, the high-dose ketamine group, while impaired, were still performing well above chance levels on the source memory task. Many previous studies have found recognition memory to be at chance following a similar dose of ketamine to that used in this study (eg Malhotra et al, 1996; Hetem et al, 2000). In these studies, ketamine disrupted encoding but not retrieval processes. The elaborative encoding procedure used in this task, where source was associated with both gender of voice and subsequent semantic judgment, appeared to elevate source memory scores predrug to near-ceiling levels. This elaborative encoding, postdrug, may also have acted to compensate partially for ketamine-induced encoding impairments.

Working memory was dose dependently impaired in the present study, accompanied by a preservation of sustained attention. This finding replicates the work of Adler et al (1998). Preserved attentional processes following ketamine administration eliminate the possibility that memory impairments are due to any general impairment in attentional functioning. It is also important to remember, when considering all the cognitive findings of the current study, that no one task taps a single memory system, for example the impaired working memory observed here may also be partially responsible for the deficits on the episodic memory task.

Our findings provide the first demonstration that perceptual priming is preserved following ketamine administration. The LOP manipulation was successful and 
demonstrated a dissociation in performance on the implicit and explicit aspects of the task, satisfying Schacter et al, (1989) 'retrieval intentionality criterion'. Thus, on wordstem completion, LOP did not affect performance, but on cued recall it did, with semantically encoded words being better remembered than physically encoded words. There was a trend towards lower scores in the ketamine groups on the cued recall task in line with previous research demonstrating that cued recall is less sensitive to druginduced impairment than free recall (Bishop and Curran, 1995). Interestingly, levels of cued recall were lower than stem completion throughout, but lowest in the high-dose ketamine group where twice as many stems were completed with targets in the implicit compared to the explicit task. This would accord with the suggestion that in situations where conscious awareness is impaired, it may serve to have inhibitory effects on explicit memory (Danion et al 1999). Preserved perceptual priming is of interest as no previous study has examined the effects of ketamine on this form of memory. This implies that a specific pattern of memory deficits is induced by ketamine rather than a global impairment on all memory tasks. Further investigation of priming with tasks such as process dissociation, which taps awareness in addition to priming, may be useful in interpreting these findings further.

No procedural learning was observed in the high-dose ketamine group and was only marginally evident in the lowdose ketamine group. In the context of other ketamine research, this relates to only one study that found an impairment in the learning of new rules, but not the expression of previously learnt rules on repeated versions of the WCST (Krystal et al, 2000). Krystal et al (2000) hypothesized that this may have been a result of deficits in the acquisition of procedural learning. However, the WCST is not a procedural measure. The findings of the present study from a task that clearly assesses procedural learning support the above hypothesis. A possible mechanism for ketamine-induced impairments on this task may stem from the putative neuroanatomical basis of procedural learning in the basal ganglia. Glutamate and the NMDA-R are essential factors in the plasticity response of the basalganglia (Schmidt, 1998) and glutamatergic activity is secondary only to dopaminergic activity in these structures (Nieoullon, 2002). As NMDA antagonism enhances both glutamate and dopamine release in components of corticostriato-thalamic circuitry (Moghaddam et al, 1997), it is feasible that this enhancement is responsible for the impairment in procedural learning observed in the current study. Previous research has found that administration of a dopamine antagonist can reverse ketamine-induced impairments on the WCST, which has a procedural learning component (Krystal et al, 1999). However, animal studies have found that working memory deficits following NMDA antagonists are more transient than increases in the prefrontal cortex and nucleus accumbens dopamine levels (Adams and Moghaddam, 1998). Therefore, further work should examine the relative contributions of glutamatergic and dopaminergic changes to this learning deficit.

It is interesting to note that even though both priming and procedural learning tap processes that do not involve conscious awareness, different findings were observed on these tasks. In patients with organic memory disorders, such as the amnesias, nonconscious forms of memory are generally preserved. The finding of impaired procedural learning in the presence of preserved priming observed in the current study, is reminiscent of the cognitive pattern observed in Parkinson's Disease (Jackson et al, 1995). However, evidence for a true impairment to procedural learning is unclear, as the RTs of the ketamine subjects were significantly slower than those of placebo overall. The speed of responding may putatively affect learning; slower responses may mean that participants require longer time to learn the sequence. Ketamine also reduced psychomotor speed on trailmaking in the present study, but did not affect trailmaking scores once psychomotor slowing had been controlled for. This replicates the findings of Harborne et al (1996), and indicates a preservation of some aspects of frontal functioning.

Retrieval from semantic memory was examined in the present study using the speed of the comprehension task. There was a dose-response relationship between the number of sentences verified on this task. This may suggest semantic memory impairments following ketamine administration. However, participants did not make any more errors after ketamine. In light of the trailmaking results discussed above and those stemming from the SRT, where RTs and speed were significantly slower in the ketamine group, it is again possible that ketamine effects on this task are due to general psychomotor slowing attributable to the anesthetic and sedative effects of the drug.

The current study replicated the findings of previous work in revealing an increase in the schizophrenic-like and dissociative symptoms following ketamine. These findings confirmed that the psychotomimetic effects of ketamine are detectable on both clinician and self-rated scales, and that ketamine, in the doses administered in this study, induced a state resembling some of the symptoms of schizophrenia. There have been suggestions in the literature that, as NMDA antagonists induce psychotic symptoms and the NMDA receptor is involved in memory, the cognitive deficits observed in schizophrenia may be mediated by the NMDA receptor (eg Newcomer and Krystal, 2001). While cognitive impairment in schizophrenia still remains ill-defined, highly tentatively it appears that the profile of cognitive effects of ketamine appears somewhat similar to that observed in schizophrenia and organic psychosis (Perlstein et al, 2001), but differs in impairments to procedural learning and preserved executive functioning. Further research using techniques such as pharmacological functional magnetic resonance imaging may shed light on the similarity between neuroanatomical substrates of NMDA antagonist-induced cognitive impairments and those observed in schizophrenia.

This study was subject to several limitations. Firstly, the cognitive testing began after $20 \mathrm{~min}$, when ketamine may not have reached a plasma level comparable to that of previous ketamine research. This could explain the lack of errors on the speed of comprehension task, as this task was administered first. Secondly, the continuous infusion style used meant that ketamine levels may have been rising throughout the study, which could result in impairments on certain tasks being an effect of increasing dose rather than ketamine specificity for certain memory systems. Studies using steady-state or pseudosteady-state infusion styles 
have negated this problem (Hetem et al, 2000; Newcomer et al, 1999). A consequence of rising blood ketamine levels may also have been that sedation was increasing, which would have compounded the effects of ketamine on these tasks. However, the data would seem to indicate that this is not the case. The tasks that were not affected by ketamine were towards the end of the battery, for example, implicit memory, when ketamine levels and drowsiness had increased. Furthermore, tasks at the beginning of the battery demonstrated ketamine-induced impairments (eg procedural learning). Thus it would appear that the selectivity of ketamine's effects on certain memory systems is not as a result of a test order that meant that tasks towards the end of the battery were subject to a combination of higher drowsiness and ketamine blood levels. Another limitation common to studies of this kind is that tasks may not be of comparable difficulty and while on tasks such as the $N$-back it is possible to manipulate levels of difficulty, on the other tasks this was not feasible. Despite this, these tasks have been shown to be differentially sensitive to the impact of pharmacological manipulations of memory systems, although further work could address this issue.

The doses of ketamine used in this experiment are somewhat lower and involved a different infusion style (continuous rather than a bolus then maintenance infusion) than some used in previous studies (eg Krystal et al, 1994; Malhotra et al, 1996; Newcomer et al, 1999). Hence comparisons across these studies are limited. Despite this, however, the cognitive effects of ketamine observed in the current study were similar to those detailed in the aforementioned papers. As this study has demonstrated discernible ketamine effects at these doses, future research may consider using lower doses, although targeting a steady state, to further investigate memory systems and ketamine. There were no baseline differences between the groups, which confirms that variation between the groups postinfusion is a result of drug effects and not individual differences. Previous studies have used crossover designs but as ketamine is associated with tachyphylaxis and potential residual cognitive effects were possible, then the independent groups design was considered to be preferable.

In summary, the present study replicated previous data, suggesting a ketamine-induced impairment of working memory and preservation of attention and executive function. This study extended findings of an impairment in episodic memory following ketamine by demonstrating an impairment in memory for source. Ketamine produced a slowing of semantic processing but no increases in errors. Further novel findings from this study were of preserved perceptual priming and impaired procedural learning induced by ketamine. This is a cognitive profile that differs from both schizophrenia and organic memory disorders.

\section{ACKNOWLEDGEMENTS}

CJAM was supported by a Medical Research Council (UK) studentship. This study was aided by a grant to HVC from the University of London UK Central Research Fund. We thank David Shanks for providing the serial reaction time task.

\section{REFERENCES}

Adams B, Moghaddam B (1998). Corticolimbic dopamine neurotransmission is temporally dissociated from the cognitive and locomotor effects of phencyclidine. J Neurosci 18: 5545-5554.

Adler CM, Goldberg TE, Malhotra AK, Breier A (1998). Effects of ketamine on thought disorder, working memory and semantic memory in healthy volunteers. Biol Psychiatry 43: 811-816.

Baddeley A (1998). Recent developments in working memory. Curr Opin Neurobiol 8: 234-238.

Baddeley AD, Emslie H, Nimmo-Smith I (1992). The speed and capacity of language processing (SCOLP) test. Bury St. Edmonds, Thames Valley Test Company.

Baddeley A, Emslie H, Nimmo-Smith I (1993). The spot the word test: a robust estimate of verbal intelligence based on lexical decision. Br J Clin Psychol 32: 55-65.

Beck AT (1978). The Beck Depression Inventory (BDI). The Psychological Corporation, Harcourt Brace Jovanovitz Inc., USA.

Bernstein E, Putnam F W (1986). Development, reliability and validity of a dissociation scale. J Nerv Mental Dis 174: 727-735.

Bishop KI, Curran HV (1995). Psychopharmacological analysis of implicit and explicit memory: a study with lorazepam and the benzodiazepine antagonist flumazenil. Psychopharmacology 121: 267-278.

Bond AJ, Lader MH (1974). The use of analogue scales in rating subjective feelings. Br J Med Psychol 47: 211-218.

Braver TS, Cohen JD, Nystrom LE, Jonides J, Smith EE, Noll DC et al (1997). A parametric study of prefrontal cortex involvement in human working memory. NeuroImage 5: 49-62.

Breier A, Malhotra AK, Pinals DA, Weisenfeld BSE, Pickar D (1997). Association of ketamine-induced psychosis with focal activation of the prefrontal cortex in healthy volunteers. Am J Psychiatry 154: 805-811.

Bremner JD, Krystal JD, Putman FW, Southwick SM, Marmar C, Charney DS et al (1998). Measurement of dissociative states with the Clinician Administered Dissociative States Scale (CADDS). J Traum Stress 11: 125-136.

Buckner RL (2001). The neuroimaging of memory. In: Gazzaniga $\mathrm{M}$ (ed). The New Cognitive Neurosciences. MIT Press: Cambridge, MA. pp 817-828.

Curran HV, Morgan CJA (2000). Cognitive, dissociative and psychotogenic effects of ketamine on recreational users on the night of drug use and 3 days later. Addiction 95: 575-590.

Danion JM, Rizzo L, Bruant A (1999). Functional mechanisms underlying impaired recognition memory and conscious awareness in patients with schizophrenia. Arch Gen Psychiatry 56: 639-644.

Ghoneim M, Hinrichs JV, Mewaldt SP, Peterson RC (1985). Ketamine: behavioural effects at subanesthetic doses. J Clin Psychopharmacol 5: 70-77.

Harborne GC, Watson FL, Healy DT, Groves L (1996). The effects of sub-anaesthetic doses of ketamine on memory, cognitive performance and subjective experience in healthy volunteers. J Psychopharmacol 10: 134-140.

Harris JA, Biersner RJ, Edwards D, Bailey LW (1975). Attention, learning and personality during ketamine emergence: a pilot study. Anesthesia Analgesia 54: 169-172.

Hetem LAB, Danion JM, Diemunsch P, Brandt C (2000). Effect of a subanesthetic dose of ketamine on memory and conscious awareness in healthy volunteers. Psychopharmacology 152: 283-288.

Jackson GM, Jackson SR, Harrison J, Henderson L, Kennard C (1995). Serial reaction time learning and Parkinson's disease: evidence for a procedural learning deficit. Neuropsychologia 33: 577-593.

Krystal JH, Belger A, D’Souza DC, Anand A, Charney DS, Aghajanian GK et al (1999). Therapeutic implications of the hyperglutamatergic effects of NMDA antagonists. Neuropsychopharmacology 21: S143-S157. 
Krystal JH, Bennett A, Abi-Saab D, Belger A, Karper LP, D’Souza DC et al (2000). Dissociation of ketamine effects on rule acquisition and rule implementation: possible relevance to NMDA receptor contributions to executive cognitive functions. Biol Psychiatry 47: 137-143.

Krystal JH, Karper LP, Bennett A, D'Souza DC, Abi-Dargham A, Morrisey $\mathrm{K}$ et al (1998). Interactive effects of subanesthetic ketamine and subhypnotic lorazepam in humans. Psychopharmacology 135: 213-299.

Krystal JH, Karper LP, Seibyl JP, Freeman GK, Delaney R, Bremner JD et al (1994). Subanesthetic effects of the non-competitive NMDA-antagonist, ketamine, in humans. Arch Gen Psychiatry 51: 199-214.

Lisman JE, Fellous J, Wang X (1998). A role for NMDA-receptor channels in working memory. Nat Neurosci 1: 273-275.

Malhotra AK, Pinals DA, Weingartner H, Sirocco K, Missar CD, Pickar D et al (1996). NMDA receptor function and human cognition: the effects of ketamine in healthy volunteers. Neuropsychopharmacology 14: 301-307.

Moghaddam B, Adams B, Verma A, Daly D (1997). Activation of glutamatergic neurotransmission by ketamine: A novel step in the pathway from NMDA receptor blockade to dopaminergic and cognitive disruptions associated with the prefrontal cortex. J Neurosci 17: 2921-2927.

Morgan CJA, Mofeez A, Brandner B, Bromley L, Curran HV (2003). Ketamine impairs response inhibition and is positively reinforcing in healthy volunteers: a dose response study. Psychopharmacology, in press.

Morris RGM, Hagan JJ, Rawlins JNP (1986). Selective impairment of learning and blockade of long-term potentiation by an $\mathrm{N}$-methyl-D-aspartate antagonist, AP5. Nature 319: 774-776.

Newcomer JW, Farber NB, Jevtovic-Todorovic V, Selke G, Melson AK, Hershey T et al (1999). Ketamine-induced NMDA receptor hypofunction as a model of memory impairment and psychosis. Neuropsychopharmacology 20: 106-118.

Newcomer JW, Krystal JH (2001). NMDA receptor regulation of memory and behaviour in humans. Hippocampus 11: 529-542.

Nieoullon A (2002). Dopamine and the regulation of cognition and attention. Prog Neurobiol 67: 53-83.

Pendelton MG, Heaton RK, Lehman RA, Hulihan D (1982). Diagnostic utility of the Thurstone word fluency test in neuropsychological evaluations. J Clin Neuropsychol 4: 307-317.

Perlstein WM, Carter CS, Noll DC, Cohen JD (2001). Relation of prefrontal cortex dysfunction to working memory and symptoms in schizophrenia. Am J Psychiatry 158: 1105-1113.

Reitan R (1958). Validity of the trail making test as an indication of organic brain damage. Percept Motor Skills 8: 271-276.

Schacter DL, Bowers J, Booker J (1989). Intention, awareness, and implicit memory: the retrieval intentionality criterion. In: Lewandowsky S, Dunn JC, Kirsner K (eds). Implicit Memory: Theoretical Issues. Erlbaum, Hillsadle, NJ. pp 47-65.

Schmidt WJ (1998). Dopamine-glutamate interactions in the basal ganglia. Amino Acids 14: 5-10.

Shanks DR, Perruchet P (2002). Dissociation between priming and recognition in the expression of sequential knowledge. Psychonomic Bull Rev 9: 362-367.

Snodgrass JG, Corwin J (1988). Pragmatics of measuring recognition memory: applications to dementia and amnesia. $J$ Exp Psychol-General 117: 34-50.

Spielberger C 1983. State-trait anxiety inventory (form Y). Palo Alto, California, Mind Garden.

Tulving E (1972). Episodic and semantic memory. In: Tulving E, Donaldson W (eds). Organization of Memory. Academic Press: New York. pp 381-403.

Tulving E (1983). Elements of Episodic Memory. Clarendon Press: Oxford.

Tulving E (1985). How many memory systems are there? Am Psychol 40: 398.

Tulving E (1998). Neurocognitive processes of human memory. In: von Euler C, Lundbreg I, Llinas R (eds). Basic Mechanisms in Cognition and Language. Elsevier: Amsterdam. pp 261-281.

Vollenweider FX, Leenders KL, Scharfetter C, Antonin A, MaGuire P, Missimer J et al (1997). Metabolic hyperfrontality and psychopathology in ketamine model of psychosis using positron emission tomography (PET) and 18F fluorodeoxyglucose. Eur Neuropsychopharmacol 7: 9-24.

Wheeler MA, Stuss DT, Tulving E (1997). Toward a theory of episodic memory: the frontal lobes and autonoetic consciousness. Psychol Bull 121: 331-354.

Wilding EL, Rugg MD (1996). An event-related potential study of recognition memory with and without retrieval of source. Brain 119: 889-905. 